\title{
Uniform discretizations: a new approach for the quantization of totally constrained systems
}

\author{
Miguel Campiglia ${ }^{1}$, Cayetano Di Bartolo ${ }^{2}$ Rodolfo Gambini ${ }^{1}$, Jorge Pullin ${ }^{3}$ \\ 1. Instituto de Física, Facultad de Ciencias, Iguá 4225, esq. Mataojo, Montevideo, Uruguay. \\ 2. Departamento de Física, Universidad Simón Bolivar, Aptdo. 89000, Caracas 1080-A, Venezuela. \\ 3. Department of Physics and Astronomy, Louisiana State University, Baton Rouge, LA 70803-4001
}

\begin{abstract}
We discuss in detail the uniform discretization approach to the quantization of totally constrained theories. This approach allows to construct the continuum theory of interest as a well defined, controlled, limit of well behaved discrete theories. We work out several finite dimensional examples that exhibit behaviors expected to be of importance in the quantization of gravity. We also work out the case of BF theory. At the time of quantization, one can take two points of view. The technique can be used to define, upon taking the continuum limit, the space of physical states of the continuum constrained theory of interest. In particular we show in models that it agrees with the group averaging procedure when the latter exists. The technique can also be used to compute, at the discrete level, conditional probabilities and the introduction of a relational time. Upon taking the continuum limit one can show that one reproduces results obtained by the use of evolving constants, and therefore recover all physical predictions of the continuum theory. This second point of view can also be used as a paradigm to deal with cases where the continuum limit does not exist. There one would have discrete theories that at least at certain scales reproduce the semiclassical properties of the theory of interest. In this way the approach can be viewed as a generalization of the Dirac quantization procedure that can handle situations where the latter fails.
\end{abstract}

\section{INTRODUCTION}

Loop quantum gravity has emerged in recent years as one of the major candidates for a theory of quantum gravity. See [1] for recent reviews. The theory has a mathematically rigorous basis for its quantum kinematics [2], which has also been proven to be unique up to certain assumptions [3]. It also has achieved attractive physical results in the context of providing a detailed microscopic picture of black hole entropy and a detailed picture of the big bang in the context of homogeneous quantum cosmologies. However, the problem of the dynamics of the full theory has remained unsettled. Related to this is the lack of a good understanding of how general relativity arises as a semiclassical limit of the quantum theory and the fact that the algebra of quantum constraints - though free of anomalies - does not mimic the algebra of the classical constraints completely.

The reason for the elusive nature of the dynamics has a well defined but technical explanation. In the accepted and largely unique kinematical setup in terms of spin networks there is a natural action of the diffeomorphism group that is a unitary representation and therefore the spatial diffeomorphisms are represented without anomalies. However, the action is not weakly continuous. This means that the infinitesimal generators of the diffeomorphism group, that is, the associated Lie algebra, cannot be defined on the Hilbert space. In contrast, versions of the Hamiltonian constraint can be defined on the Hilbert space. However, since the classical Poisson algebra of constraints involves the infinitesimal generators of diffeomorphisms, it should not be surprising that the classical constraint algebra is not appropriately reflected in the quantum constraint algebra. A clear discussion of this problem is present in the introduction to the recent paper by Giesel and Thiemann [4]. There does not appear to exist an obvious solution to this problem in the sense of constructing a "better" Hamiltonian constraint operator, for obvious reasons. This obstruction has led several researchers to consider alternatives to the usual Dirac approach to the problem of the dynamics. One of the alternatives is the "master constraint" project of Thiemann and collaborators [5] motivated by an earlier proposal by Klauder [6]. Others consider the covariant "spin foam" approach as an alternative, since one may bypass the construction of the canonical algebra entirely, at least in some settings. Another approach is the one furthered in this paper, which we will refer to as "uniform discretizations".

Over the last few years we have developed a new paradigm for dealing with totally constrained theories (see [7, [8, 9 ] for recent reviews). The approach has been called "consistent discretizations" and a particularly promising version of it recently introduced [10] is called "uniform discretizations". To describe the paradigm in a nutshell one can say that its relationship to totally constrained theories is the same that lattice QCD has with continuum QCD. The new approach consists in building a set of discrete theories and to define a continuum theory as a suitably well defined limit. Just like one can view lattice QCD as a tool to define continuum QCD non-perturbatively one can view the uniform discretization as a tool to define continuum theories that are totally parameterized, as general relativity. Therefore the goal is to have a discretized version of the theory that is well defined, under control and quantizable without difficulties and only then study how to take the continuum limit. Other approaches to the dynamics of 
quantum gravity currently being considered also use discretizations, but the continuum limit is immediately taken since the discrete theories are in themselves inconsistent, i.e. they do not exist.

The naive application of a discretization - like the ones used in lattice QCD - to gravity or other totally constrained field theories immediately fails since the introduction of the lattice conflicts with diffeomorphism invariance. From an operational viewpoint, this translates itself in the failure of constraint algebras to close. In other words, the discretization of a totally constrained theory generically yields a discrete theory that is inconsistent, its equations cannot be simultaneously solved. This is manifested by the lack of closure of constraint algebras (more precisely, the constraints do not structure themselves into an algebra). A typical example of such an approach is the one followed in numerical relativity in the Cauchy approach, where it is well known that one cannot solve simultaneously the evolution equations and the constraint equations. In that context the usual approach is to ignore some of the equations - typically the constraints - in the hope that if one starts with data that satisfies them initially, they will remain small upon evolution. The logic is an a-posteriori one, that is, if one carries out a simulation and the constraints remain small, then it is an acceptable approximation to the continuum theory. But it frequently happens that they do not remain small and the simulation then has to be abandoned. Much more serious is the situation if one is interested in quantizing. To use a set of inconsistent theories to define via a limiting process a continuum theory at the quantum level is clearly not logically tenable; to exist a limit one first has to have a sequence. In quantum mechanics one cannot just pick and choose some initial data and hope that "constraints will remain small". Since the inconsistent theories do not exist, their quantization makes no sense and one cannot construct a sensible continuum limit.

This is the reason why we have been studying the possibility of constructing discretizations of totally covariant theories that are consistent, that is, the equations of the discrete theory can be solved simultaneously. How can this be achieved? Largely by turning the quantities that in the continuum play the role of Lagrange multipliers into dynamical variables. The extra variables allow to make compatible the set of equations that were formerly incompatible. For instance, in general relativity written in terms of metric variables in the traditional $3+1$ decomposition, one has twelve canonical variables and twelve evolution equations, plus four constraint equations. One has therefore 16 equations for 12 unknowns. In the continuum the equations are compatible and can be solved. When discretized however, one ends up with a set of incompatible equations. If we now consider the three components of the shift and the lapse as dynamical variables we have 16 equations with 16 dynamical variables as unknowns and simultaneous solution of the equations is generically possible.

An immediate question that arises is: if the Lagrange multipliers are now determined, is this not equivalent to choosing a gauge? The answer is obviously negative, since the resulting theory has more degrees of freedom than the one we started from, it cannot simply be a gauge fixing of it. The coordinate freedom is indeed encoded in the extra degrees of freedom of the discrete theory. Still, since the Lagrange multipliers are determined (and in the case of a totally constrained theory this means the lapse is determined), how is the continuum limit to be materialized? The answer is that one can approximate the continuum theory by choosing initial data in the discrete theory in a careful way. To put it differently there exist initial data for the discrete theory such that when evolved the resulting solution approximates well the solutions of the continuum theory. There also exist initial data for the discrete theory that do not approximate the continuum at all. The continuum limit therefore is defined by choosing suitably limits within families of initial data. To do this in a consistent way within a canonical framework and throughout all of phase space is quite non-trivial. This is what the "uniform discretizations" achieve. There one discretizes the theory in a way that certain quantities are conserved upon evolution and therefore one has a well defined way to characterize the continuum limit to be taken in terms of the initial data.

The uniform discretizations were introduced briefly in reference [10]. The purpose of this paper is to apply the uniform discretization technique to a set of examples of increasing level of challenge. Most of the examples are the same ones as Thiemann and collaborators have considered in their master constraint program. The organization of the paper is as follows: in section 2 we briefly review the consistent and uniform discretization techniques, in section 3 we consider the first example: homogeneous cosmologies; in section 4 we consider a model with Abelian constraints, in section 5 a model with non-Abelian constraints that structure themselves into a Lie algebra, in section 6 we consider a system with a non-Abelian and non-compact Lie algebra of constraints, in section 7 we explore BF theory as a first example of a field theory. We end with a discussion. The examples of section 4, 5, 6 have been discussed in detail with the "master constraint" approach [5]. 


\section{CONSISTENT AND UNIFORM DISCRETIZATIONS: A BRIEF REVIEW}

\section{A. Consistent discretizations}

We start by considering a continuum theory representing a mechanical system. Its Lagrangian will be denoted by $\hat{L}\left(q^{a}, \dot{q}^{a}\right), a=1 \ldots M$. This setting is general enough to accommodate, for instance, totally constrained systems. In such case $\dot{q}$ will be the derivative of the canonical variables with respect to the evolution parameter. It is also general enough to include the systems that result from formulating on a discrete space-time lattice a continuum field theory, like general relativity.

We discretize the evolution parameter in intervals (possibly varying upon evolution) $t_{n+1}-t_{n}=\epsilon_{n}$ and we label the generalized coordinates evaluated at $t_{n}$ as $q_{n}$. We define the discretized Lagrangian as

$$
L(n, n+1) \equiv L\left(q_{n}^{a}, q_{n+1}^{a}\right) \equiv \epsilon_{n} \hat{L}\left(q^{a}, \dot{q}^{a}\right)
$$

where

$$
q^{a}=q_{n}^{a} \quad \text { and } \quad \dot{q}^{a} \equiv \frac{q_{n+1}^{a}-q_{n}^{a}}{\epsilon_{n}} .
$$

Of course, one could have chosen to discretize things in a different fashion, for instance using a different approximation for the derivative, or by choosing to write the continuum Lagrangian in terms of different variables. The resulting discrete theories generically will be different and will approximate the continuum theory in different ways. However, given a discrete theory, the treatment we outline is unique. We will take advantage of this freedom in how to discretize the theory to propose the "uniform discretizations" in the next section.

The action can then be written as

$$
S=\sum_{n=0}^{N} L(n, n+1) .
$$

It should be noted that in previous treatments we have sometimes written the Lagrangian in first order form, i.e. $L=\int d t(p \dot{q}-H(p, q))$. It should be emphasized that this is contained as a particular case in the treatment we are presenting here. In this case one takes both $q$ and $p$ to be configuration variables, and one is faced with a Lagrangian that involves $q_{n}, p_{n}$ and $q_{n+1}$ as variables and does not depend on $p_{n+1}$. The reason we frequently resort to first order formulations in the various concrete examples we discuss is that the Ashtekar formulation used in loop quantum gravity is naturally a first order one and we usually tend to frame things in a closely related way. But again, there is no obstruction to using either first or second order formulations with our framework, they are both contained as particular cases.

If the continuum theory is invariant under reparameterizations of the evolution parameter, one can show that the information about the intervals $\epsilon_{n}$ may be absorbed in the Lagrange multipliers. In the case of standard mechanical systems it is simpler to use an invariant interval $\epsilon_{n}=\epsilon$.

The Lagrange equations of motion are obtained by requiring the action to be stationary under variations of the configuration variables $q^{a}$ fixed at the endpoints of the evolution interval $n=0, n=N+1$,

$$
\frac{\partial L(n, n+1)}{\partial q_{n}^{a}}+\frac{\partial L(n-1, n)}{\partial q_{n}^{a}}=0 .
$$

We introduce the following definition of the canonically conjugate momenta of the configuration variables,

$$
\begin{aligned}
p_{n+1}^{a} & \equiv \frac{\partial L(n, n+1)}{\partial q_{n+1}^{a}} \\
p_{n}^{a} & \equiv \frac{\partial L(n-1, n)}{\partial q_{n}^{a}}=-\frac{\partial L(n, n+1)}{\partial q_{n}^{a}}
\end{aligned}
$$

Where we have used Eq. (4). The equations (5) and (6) define a canonical transformation for the variables $q_{n}, p_{n}$ to $q_{n+1}, p_{n+1}$ with a the type 1 generating function $F_{1}=-L\left(q_{n}^{a}, q_{n+1}^{a}\right)$. Notice that the evolution scheme is implicit, one can use the bottom equation (since we are in the non-singular case) to give an expression for $q_{n+1}$ in terms of $q_{n}, p_{n}$, which in turn can be substituted in the top equation to get an equation for $p_{n+1}$ purely in terms of $q_{n}, p_{n}$.

It should be noted that there are several other possible choices, when going from the set of equations (516) to an explicit evolution scheme (see Di Bartolo et al. [11] for further details.) 
The canonical transformation we introduced becomes singular as an evolution scheme if $\left|\frac{\partial^{2} L(n, n+1)}{\partial q_{n+1}^{a} \partial q_{n}^{b}}\right|$ vanishes. If the rank of the matrix of second partial derivatives is $K$ the system will have $2(M-K)$ constraints of the form,

$$
\begin{aligned}
\Phi_{A}\left(q_{n}^{a}, p_{n}^{a}\right) & =0 \\
\Psi_{A}\left(q_{n+1}^{a}, p_{n+1}^{a}\right) & =0 .
\end{aligned}
$$

And these constraints need to be enforced during evolution, which may lead to new constraints. We refer the reader for the detailed Dirac analysis to Di Bartolo et al. [11].

To clarify ideas, let us consider an example. The model consists of a parameterized free particle in a two dimensional space-time under the influence of a linear potential. The discrete Lagrangian is given by,

$$
\begin{aligned}
L_{n} & \equiv L\left(q_{n}^{a}, \pi_{n}^{a}, N_{n}, q_{n+1}^{a}, \pi_{n+1}^{a}, N_{n+1}\right) \\
& =\pi_{n}^{a}\left(q_{n+1}^{a}-q_{n}^{a}\right)-N_{n}\left[\pi_{n}^{0}+\frac{1}{2}\left(\pi_{n}^{1}\right)^{2}+\alpha q_{n}^{1}\right] .
\end{aligned}
$$

We have chosen a first order formulation for the particle (otherwise there are no constraints and the example is trivial). However, this Lagrangian is of the type we considered in this presentation, one simply needs to consider all variables, $q^{a}, \pi^{a}, N$ as configuration variables. The system is clearly singular since the $\pi^{\prime} s$ and $N$ only appear at level $n$ (or in the continuum Lagrangian, their time derivatives are absent). When considered as a Type I generating function, the above Lagrangian leads to the equations

$$
\begin{aligned}
p_{\pi, n+1}^{a} & =\frac{\partial L_{n}}{\partial \pi_{n+1}^{a}}=0 \\
p_{q, n+1}^{a} & =\frac{\partial L_{n}}{\partial q_{n+1}^{a}}=\pi_{n}^{a} \\
p_{N, n+1} & =\frac{\partial L_{n}}{\partial N_{n+1}}=0
\end{aligned}
$$

and

$$
\begin{aligned}
& p_{\pi, n}^{a}=-\frac{\partial L_{n}}{\partial \pi_{n}^{a}}=-\left(q_{n+1}^{a}-q_{n}^{a}\right)+\pi_{n}^{1} N_{n} \delta_{1}^{a}+N_{n} \delta_{0}^{a}, \\
& p_{q, n}^{a}=-\frac{\partial L_{n}}{\partial q_{n}^{a}}=\pi_{n}^{a}+\delta_{1}^{a} \alpha N_{n}, \\
& p_{N, n}=-\frac{\partial L_{n}}{\partial N_{n}}=\pi_{n}^{0}+\frac{1}{2}\left(\pi_{n}^{1}\right)^{2}+\alpha q_{n}^{1} .
\end{aligned}
$$

The constraints (10[12]14]15) can be imposed strongly to eliminate the $\pi$ 's and the $N$ 's and obtain an explicit evolution scheme for the $q$ 's and the $p_{q}$ 's,

$$
\begin{aligned}
q_{n}^{0} & =q_{n+1}^{0}-\frac{C_{n+1}}{\alpha p_{q, n+1}^{1}}, \\
q_{n}^{1} & =q_{n+1}^{1}-\frac{C_{n+1}}{\alpha}, \\
p_{q, n}^{0} & =p_{q, n+1}^{0}, \\
p_{q, n}^{1} & =p_{q, n+1}^{1}+\frac{C_{n+1}}{p_{q, n+1}^{1}},
\end{aligned}
$$

and the Lagrange multipliers get determined to be,

$$
N_{n}=\frac{C_{n+1}}{\alpha p_{q, n+1}^{1}}
$$

where $C_{n+1}=p_{q, n+1}^{0}+\left(p_{q, n+1}^{1}\right)^{2} / 2+\alpha q_{n+1}^{1}$. The evolution scheme runs backwards, one can construct a scheme that runs forward by solving for $N$ and $\pi$ at instant $n$ when imposing the constraints strongly. The two methods yield evolution schemes of different functional form since one propagates "forward" in time and the other "backward". The 
inequivalence in the functional form stems from the fact that the discretization of the time derivatives chosen in the Lagrangian is not centered. It should be emphasized that if one starts from given initial data and propagates forwards with the first system of equations and then backwards using the second, one will return to the same initial data.

So we see in the example how the mechanism works. It yields evolution equations that usually are implicit as evolution schemes. The equations are consistent. The Lagrange multipliers get determined by the scheme and there are no constraints left on the canonical variables. The evolution is implemented by a (non-singular) canonical transformation. The number of degrees of freedom is larger than those in the continuum. There will exist different sets of initial data that lead to different solutions for the discrete theory but nevertheless will just correspond to different discrete approximations and parameterizations of a single evolution of the continuum theory.

The example also exhibits some of the problems the framework may face. Since one is generically left with implicit non-linear systems of algebraic equations to be solved, there is no guarantee that the solutions will be real. Neither is it guaranteed that the Lagrange multipliers will be bounded in value (this is important since in totally constrained theories the lapse controls the evolution step and one wishes it to be small in the continuum limit). Although we explored some simple examples where this does not pose a problem in several publications, it is clear that generically it could be a serious obstacle. This obstacle can be related to the intuitive fear that people manifested about the formalism being a "gauge fixing". Gauge fixed quantizations are not favored since it is known that one cannot fix gauges globally. Similarly here, the framework cannot generically guarantee that one approximates the continuum globally in a solution. We will now argue that some simple, yet carefully chosen, discretizations actually achieve this goal. This is in principle achieved in a generic fashion, i.e. we can find such discretizations for any given totally constrained system.

\section{B. Uniform discretizations: the key idea}

As we stressed before, there is significant freedom in how one chooses to discretize the action. We will now use this freedom to our advantage, as was first proposed in [10]. Consider a totally constrained system with $N$ first class constraints $\phi_{i}$ and a configuration space with $2 P$ variables $(q, p)$ (we omit indices on the variables for simplicity). We will assume that one has chosen the discretization of the action in such a way that the evolution equations for a given dynamical variable $A$ of the theory can be written as,

$$
A_{n+1}=e^{\{\bullet, H\}}\left(A_{n}\right) \equiv A_{n}+\left\{A_{n}, H\right\}+\frac{1}{2}\left\{\left\{A_{n}, H\right\}, H\right\}+\cdots
$$

As is obvious, $H$ is a constant of the motion of the discrete evolution. The quantity $H$ is defined in the following way: Consider a smooth function of $N$ variables $f\left(x_{1}, \ldots, x_{N}\right)$ such that the following three conditions are

satisfied: a) $f\left(x_{1}, \ldots, x_{N}\right)=0 \Longleftrightarrow x_{i}=0 \forall i$ and otherwise $f>0 ;$ b) $\frac{\partial f}{\partial x_{i}}(0, \ldots, 0)=0 ;$ c) $\operatorname{det} \frac{\partial^{2} f}{\partial x_{i} \partial x_{j}} \neq 0$ $\forall x$ and d) $f\left(\phi_{1}(q, p), \ldots \phi_{N}(q, p)\right)$ is defined for all $(q, p)$ in the complete phase space. Given this we define $H(q, p) \equiv f\left(\phi_{1}(q, p), \ldots \phi_{N}(q, p)\right)$.

A particularly simple example is $H(q, p)=1 / 2 \sum_{i=1}^{N} \phi_{i}(q, p)^{2}$, a choice that has interesting parallels with the "master constraint" of the "Phoenix project" [5] as we shall discuss later.

An important point is that if we choose initial data such that $H<\epsilon$ then $\phi_{i}$ remain bounded throughout the evolution and will tend to zero in the limit $\epsilon \rightarrow 0$. Let us see that in this limit one recovers the evolution equations given by the total Hamiltonian $H_{T}$ in the constrained continuum theory. Let $H$ as in the simple example above and take its initial value to be $H_{0}=\delta^{2} / 2$. We define $\lambda_{i}=\phi_{i} / \delta$, and therefore $\sum_{i=1}^{N} \lambda_{i}^{2}=1$. The evolution of the dynamical variable $q$ is given by,

$$
q_{n+1}=q_{n}+\sum_{i=1}^{N}\left\{q_{n}, \phi_{i}\right\} \lambda_{i} \delta+O\left(\delta^{2}\right)
$$

and if we define $\dot{q} \equiv \lim _{\delta \rightarrow 0}\left(q_{n+1}-q_{n}\right) / \delta$, where we have identified the "time evolution" step with the initial data choice for $\delta$, one then has,

$$
\dot{q}=\sum_{i=1}^{N}\left\{q, \phi_{i}\right\} \lambda_{i}
$$

and similarly for other dynamical variables. The specific values of the multipliers $\lambda_{i}$ depend on the initial values of the constraints $\phi_{i}$. Notice that taking the continuum limit requires that the Lagrange multipliers be determined as is 
usual in the consistent discretization approach, but are well defined bounded real functions of phase space, bypassing an important objection to the original approach.

Notice that the procedure deals only with first class constraints. In case of a system with second class constraints, it will be needed to use Dirac brackets and work with the equivalent first class system.

An important comment is needed here about the difference in the case of a mechanical system and a field theory. Although it is true that field theories formulated on the lattice are mechanical systems, generically the constraints of field theories formulated on the lattice fail to be first class, even if they were so in the continuum. If the constraints of the discrete theory are not first class the above procedure fails to reproduce the equations of the continuum. Here one has two choices. One would be to first take the continuum limit spatially keeping time discrete. In that case it may happen that the resulting constraints of the theory with continuum space and discrete time are first class. Then the above proof goes through. The alternative is to consider in the discrete evolution equation (22) the Dirac bracket of the discrete theory. Then one can show that the continuum limit is correctly achieved, including in the Hamiltonian both first and second class constraints.

One could recast the current proposal in terms of the original approach to consistent discretizations. There one started from an action and noted that the Lagrangian could be viewed as a the generating function of a canonical transformation between instants $n$ and $n+1$. To be concrete, let us analyze a system with $N$ Abelian constraints. We introduce the Lagrangian

$$
L\left(q_{n}, q_{n+1}, \lambda_{1}, \ldots, \lambda_{N}\right)=S\left(q_{n}, q_{n+1}, \lambda_{1}, \ldots, \lambda_{N}\right)+g\left(\lambda_{1}, \ldots, \lambda_{N}\right)
$$

where $L$ is a type 1 generating function of a canonical transformation between canonical variables $q_{n}, p_{n}$ and $q_{n+1}, p_{n+1}$, $S$ is Hamilton's principal function for a given set of Lagrange multipliers $\lambda_{1}, \ldots, \lambda_{N}$ (they are evaluated at instant $n$, we omit the subscript for simplicity), $g$ is such that $g(0)=0$ and the mappings $\lambda_{i} \rightarrow \frac{\partial g}{\partial \lambda_{i}}$ and $x_{i} \rightarrow \frac{\partial f}{\partial x_{i}}$ are inverse where $f$ is the function used to define the Hamiltonian. The generating function yields the canonical momenta in the usual way $p_{n+1}=\partial L / \partial q_{n+1}, p_{n}=-\partial L / \partial q_{n}$. One also has that $\partial L / \partial \lambda_{i}=0$ and this determines the Lagrange multipliers, $\lambda_{i}=h_{i}(\phi)$, where $h_{i}$ is the inverse function of the mapping defined by $\lambda_{i} \rightarrow \frac{\partial g}{\partial \lambda_{i}}$. This evolution corresponds to a Hamiltonian $H=f\left(\phi_{1}, \ldots, \phi_{N}\right)$, with $\partial_{i} f=h_{i}$. In particular, the simplest case is when $g=\sum_{i=1}^{N} x_{i}^{2} / 2$ and then $H=\sum_{i=1}^{N} \phi_{i}^{2} / 2$. The generating function $L$ allows to determine the discrete evolution that preserves exactly the value of the constraints of the continuum theory and recovers the continuum limit when all $\phi_{i} \rightarrow 0$ in the initial data. We therefore see that the approach proposed here is a particular case of the consistent discretizations. We have just chosen to discretize things in a way that the Hamiltonian is simple - rather than the action - and this guarantees a good continuum limit.

The constants of the motion of the discrete theory are quantities that have vanishing Poisson bracket with the Hamiltonian, $\left\{O_{i}^{D}, H\right\}=0$ and in the continuum limit $H_{0} \rightarrow 0$ reproduce, as functions of phase space the "perennials" of the continuum theory $O_{i}^{C}=\lim _{H_{0} \rightarrow 0} O_{i}^{D}$. This can be immediately seen from the fact that the discrete equations reproduce the continuum equations for any dynamical variable in the continuum limit. Conversely, for every perennial of the continuum theory there exists a constant of the motion (in general many constants) of the discrete theory that reduce to the given perennial in the continuum limit. We have therefore shown that uniform discretizations recover the constraints and the perennials of the continuum theory and therefore provide a good starting point for a quantization of the continuum theory.

\section{Quantization}

We now turn our attention to the quantum theory. We will introduce a Heisenberg quantization for the discrete theory (this is more natural given that one has an explicit evolution). To quantize the theory we follow several steps. We start with the classical discrete system constructed as in the previous section, we then have the canonical variables at level $n+1$ in terms of the variables at level $n, q_{n+1}=q_{n+1}\left(q_{n}, p_{n}\right), \quad p_{n+1}=p_{n+1}\left(q_{n}, p_{n}\right)$.

We then define the kinematical space of states of the quantum theory, $\mathcal{H}_{k}$, as the space of functions of $N$ real variables $\psi(q)$ that are square integrable. In this space we define operators $\hat{Q}$ and $\hat{P}$ as usual. To construct the operators at other time levels (in the Heisenberg Picture) we introduce a unitary operator $\hat{U}$ that we will define later that gives,

$$
\hat{Q}_{n} \equiv \hat{U}^{-1} \hat{Q}_{n-1} \hat{U}=\hat{U}^{-n} \hat{Q}_{0} \hat{U}^{n}, \quad \hat{P}_{n} \equiv \hat{U}^{-1} \hat{P}_{n-1} \hat{U}=\hat{U}^{-n} \hat{P}_{0} \hat{U}^{n}
$$

When the evolution is determined by a discrete Hamiltonian $H$, as is the case in the uniform discretizations, the evolution operator is given by $\hat{U}=e^{-i \hat{H} / \hbar}$. Notice that $\hat{U}$ may also be determined by requiring that the fundamental 
operators satisfy an operatorial version of the evolution equations,

$$
\hat{Q}_{n} \hat{U}-\hat{U} Q_{n+1}\left(\hat{Q}_{n}, \hat{P}_{n}\right)=0, \quad \hat{P}_{n} \hat{U}-\hat{U} P_{n+1}\left(\hat{Q}_{n}, \hat{P}_{n}\right)=0,
$$

and this provides a consistency criterion for the construction of $\hat{U}$.

At a classical level $H=0$ if and only if the constraints $\phi_{i}=0$. There exists a natural definition of the physical space of the continuum theory that does not require that we refer to the constraint. Since we know that $\hat{U}=\exp (-i \hat{H} / \hbar)$, a necessary condition satisfied by the states of the physical space of the continuum theory, $\psi \in \mathcal{H}_{\text {phys }}$ is given by $\hat{U} \psi=\psi$. More precisely the states $\psi$ of $\mathcal{H}_{\text {phys }}$ should belong to the dual of a space $\Phi$ of functions sufficiently regular on $\mathcal{H}_{k}$. That is, the states $\psi \in \mathcal{H}_{\text {phys }}$ satisfy

$$
\int \psi^{*} \hat{U}^{\dagger} \varphi d q=\int \psi^{*} \varphi d q
$$

where $\varphi \in \Phi$. This condition characterizes the quantum physical space of a constrained continuum theory without needing to implement the constraints as quantum operators by using the discretization technique.

The unitary operators of the discrete theory allow to construct the "projectors" onto the physical space of the continuum theory, which is one of the main goals of any quantization procedure based on Dirac's ideas. It should be noted that these are really generalized projectors in the sense that they project to a set of functions that belong in the dual of a subspace of sufficiently well behaved functions of $\mathcal{H}_{k}$. All of this is achieved without having to define the quantum constraint. To construct the "projectors" one can compute,

$$
\hat{P} \equiv \lim _{M \rightarrow \infty} C_{M} \hat{U}^{M} .
$$

If such a limit exists for some $C_{M}$ such that $\lim _{M \rightarrow \infty}\left(C_{M+1} / C_{M}\right)=1$ then $\hat{U} \hat{P}=\hat{P}$, and we have that $\hat{U} \hat{P} \psi=\hat{P} \psi$, $\forall \psi \in \mathcal{H}_{\mathrm{k}}$.

The limit exists in several examples in which $\hat{H}$ has a continuum spectrum, as we shall see. If the spectrum is discrete with eigenvalues $e_{i}$ and it contains a vanishing eigenvalue $e_{i_{0}}$ then a projector is trivially defined as $\left|e_{i_{0}}><e_{i_{0}}\right|$. A constructive procedure leading to a general definition of the projector in terms of the discrete evolution operator $\hat{U}$ valid for any spectrum, continuum or discrete, is given by:

$$
\hat{P} \equiv \lim _{M \rightarrow \infty} \sum_{n=M}^{\operatorname{Int}(r M)} \frac{C_{n} \hat{U}^{n}}{\operatorname{Int}(r M)-M} .
$$

where $r$ is a real number grater than one and $\operatorname{Int}(r M)$ is the integer part of $r M$. If $\hat{U}$ has a continuum spectrum this definition is a trivial consequence of the previous one. In the case of a discrete spectrum one can check that the definition works recalling the definition of the Kronecker delta in terms of a Fourier series. Notice that the definition of physical space that Thiemann introduces in the "Phoenix project" [5], is equivalent to the choice we make if one is considering the Hamiltonian that is quadratic in the constraints. Furthermore, given two states of $\mathcal{H}_{\mathrm{phys}}, \psi_{\mathrm{ph}}$, $\phi_{\mathrm{ph}}$, where $\psi_{\mathrm{ph}}=\hat{P} \psi$, and $\phi_{\mathrm{ph}}=\hat{P} \phi$, the physical inner product is defined by $<\psi_{\mathrm{ph}} \mid \phi_{\mathrm{ph}}>=\int d q \phi(q)^{*} \hat{P} \psi(q)$ and a physical inner product is determined by the projector constructed from the discrete theory.

We now illustrate the technique with a rather general example. We consider a generic mechanical system with a finite dimensional phase space with one constraint $\phi=0$. We will show that the projector constructed with our technique reproduces the one constructed with group averaging techniques [12]. That is,

$$
P=\lim _{M \rightarrow \infty} \sqrt{\frac{i M}{\pi}} e^{-i M \phi^{2}}=\int_{-\infty}^{\infty} \frac{d \mu}{2 \pi} e^{i \mu \phi} .
$$

To make contact with the group averaging case we need to assume that $\phi$ is a self-adjoint operator in the kinematical phase space with an eigenbasis given by $\phi|\alpha>=\phi(\alpha)| \alpha>$ and $1=\int|\alpha><\alpha| d \alpha$. The proof of the equivalence is,

$$
P=P \int|\alpha><\alpha| d \alpha=\int \lim _{M \rightarrow \infty} \sqrt{\frac{i M}{\pi}} e^{-i M \phi^{2}}|\alpha><\alpha|
$$

and noting that $\lim _{M \rightarrow \infty} \sqrt{\frac{i M}{\pi}} e^{-i M x^{2}}=\delta(x)=\int_{-\infty}^{\infty} \frac{d \mu}{2 \pi} e^{i \mu x}$ the proof is complete. For the proof we assumed a quadratic form of the Hamiltonian, but can actually be extended to Hamiltonians of the general form we discussed above, computing the integral by steepest descents. The proof can also be extended to systems with $N$ Abelian constraints by noting that $\lim _{M \rightarrow \infty}\left(\frac{i M}{\pi}\right)^{N / 2} e^{-i M \vec{x} \cdot \vec{x}}=\delta(\vec{x})=\int_{-\infty}^{\infty} d \mu^{N} e^{i \vec{\mu} \cdot \vec{x}} /(2 \pi)^{N}$.

A point to be noted is that if the Hamiltonian is such that zero is not an eigenvalue, the above expression for the projector vanishes. Therefore the technique cannot be applied. We will confront a situation like this in section VI. There we will argue that in such models one should take a different point of view than defining the physical Hilbert space in the traditional way. 


\section{EXAMPLE 1: HOMOGENEOUS ISOTROPIC COSMOLOGY}

We will follow the same procedure as in ordinary loop quantum cosmology (see [13] for a review). We start by considering general relativity formulated in terms of Ashtekar's variables. We then assume that we have a homogeneous and isotropic universe with flat spatial sections. In such case one can choose the triad and Ashtekar connection as,

$$
A_{a}^{i}(x)=\frac{c \delta_{a}^{i}}{V_{0}^{1 / 3}}, \quad E_{i}^{a}=\frac{p \delta_{i}^{a}}{V_{0}^{2 / 3}}
$$

with $\{c, p\}=(8 \pi / 3) G$ where we have taken the Immirzi parameter as unity. As usual one proceeds in analogy with the full theory and defines the holonomy along an "edge" of "length" $\mu V_{0}^{1 / 3}$, where $V_{0}$ is the volume an elementary cell of universe,

$$
h_{i}^{(\mu)}(c)=\cos (\mu c / 2)+2 \tau_{i} \sin (\mu c / 2) .
$$

The loop quantum cosmology approach, roughly speaking, can be characterized as attempting to produce for the homogeneous context expressions that mimic those that are found in the loop representation for the full theory. In particular, one takes the usual Hamiltonian constraint of the classical theory and represents the quantities involving connections using holonomies and the expressions involving the triads using the Poisson bracket of the volume with the connection, as first noted by Thiemann in the full theory [14].

The resulting expression for the Hamiltonian is, written as

$$
C_{\text {grav }}^{\left(\mu_{0}\right)}=-\frac{4 \operatorname{sgn}(p)}{8 \pi \mu_{0}^{3} G} \sum_{i j k} \epsilon^{i j k} \operatorname{Tr}\left(h_{i}^{\left(\mu_{0}\right)} h_{j}^{\left(\mu_{0}\right)} h_{i}^{\left(\mu_{0}\right)-1} h_{k}^{\left(\mu_{0}\right)}\left\{h_{k}^{\left(\mu_{0}\right)-1}, V\right\}\right)+O\left(c^{3} \mu_{0}\right)
$$

where $V=|p|^{3 / 2}$. In the continuum limit $\left(\mu_{0} \rightarrow 0\right)$ it can be shown that the expression reproduces the Hamiltonian constraint of a homogeneous isotropic vacuum minisuperspace.

It turns out to be more convenient to evaluate explicitly the Poisson bracket and replace the holonomies by the expression above to get,

$$
C_{\text {grav }}^{\left(\mu_{0}\right)}=-\sqrt{6} \frac{\sqrt{16 \pi G|p|}}{\mu_{0}^{2}} \sin ^{2}\left(\mu_{0} c\right)
$$

Where we have made the redefinition $\frac{3}{8 \pi G} p \rightarrow p$ so that $\{c, p\}=1$, we will use this redefinition of $p$ in what follows. This Hamiltonian constraint can be obtained from a Lagrangian for gravity coupled to a scalar field of the form,

$$
L=p \dot{c}+p_{\phi} \dot{\phi}+\frac{M}{2}\left[\frac{\sqrt{|p|} \sin ^{2}\left(\mu_{0} c\right)}{\mu_{0}^{2}}-\frac{p_{\phi}^{2}}{|p|^{3 / 2}}\right] .
$$

$M$ is the Lagrange multiplier (proportional to the lapse) with the factor $\sqrt{6} \sqrt{16 \pi G}$ absorbed in it. For simplicity we have also rescaled the scalar field and its conjugate so the gravitational coupling $3 /(16 \pi G)$ is absorbed.

We immediately recognize in the classical limit $\mu_{0} \rightarrow 0$ the usual Lagrangian for the Friedmann-Robertson-Walker model coupled to a scalar field.

Given that we are dealing with a mechanical system with one constraint, one can immediately apply the technique used in the example of the last section to show that one recovers the same physical space as in the group averaging approach. We therefore make contact exactly with traditional loop quantum cosmology, at the level of physical space.

It would be interesting to pursue this example further by not taking the continuum limit. There one could introduce a relational time and study loss of unitarity and other effects that will arise, but we will postpone a detailed discussion of cosmology to a subsequent paper.

\section{EXAMPLE 2: A MODEL WITH ABELIAN CONSTRAINTS}

We consider a mechanical system with configuration manifold $R^{N}$, coordinatized by $q^{i}, i=1, \ldots, N$ and $M<N$ commuting constraints

$$
C^{i}=p^{i} \quad i=1, \ldots, M
$$


where the $p^{i}$ 's are the conjugated momenta to the $q^{i}$ 's.

All phase space functions which do not depend on the first $M q^{i}$ 's are Dirac observables, i.e. functions which commute with the constraints (on the constraint hypersurface).

A Dirac observable which depends on $p^{i}, i=1, \ldots, M$ is equivalent to the Dirac observable obtained from the first one by setting $p^{i}=0$ (since these two observables will coincide on the constraint hypersurface). Therefore it is sufficient to consider observables which are independent of the first $M$ configuration observables and of the first $M$ conjugate momenta. A canonical choice for an observable algebra is the one generated by $q^{i}, p^{i}, i=(M+1), \ldots, N$.

Let us now consider the uniform discretization. For that we construct $H=\frac{k}{2} \sum_{i=1}^{M}\left(p^{i}\right)^{2}$. The constant $k$ is to make the Hamiltonian a dynamical variable with units of action. The discrete evolution equations are,

$$
\begin{aligned}
q_{n+1}^{a} & =q_{n}^{a}+k \sum_{b=1}^{M}\left\{q_{n}^{a}, p_{n}^{b}\right\} p_{n}^{b}, \\
& =q_{n}^{a}+k p_{n}^{a} \Theta_{a, M},(\text { there is no summation in } a) \\
p_{n+1}^{a} & =p_{n}^{a},
\end{aligned}
$$

where $\Theta_{a, M}=1$ if $a \leq M$ and zero otherwise.

To take the classical continuum limit, we assume that $H=\delta^{2} / 2$ with $\delta \rightarrow 0$. If we define

$$
\dot{q}^{a} \equiv \lim _{\delta \rightarrow 0} \frac{q_{n+1}^{a}-q_{n}}{\delta}
$$

we have from the evolution equations that,

$$
\begin{aligned}
\dot{q}^{a} & =\lambda^{a} \Theta_{a M}, \\
\dot{p}^{a} & =0
\end{aligned}
$$

where $\lambda^{a} \equiv \lim _{\delta \rightarrow 0} k p^{a} / \delta$.

To quantize the system, we will start with an auxiliary Hilbert space $L_{2}\left(R^{N}\right)$ on which the operators $\hat{q}^{i}$ act as multiplication operators and the momenta as derivatives,

$$
\begin{aligned}
& \hat{q}^{i} \psi(q)=q^{i} \psi(q) \\
& \hat{p}^{i} \psi(q)=-i \hbar \partial_{i} \psi(q),
\end{aligned}
$$

and construct a unitary operator,

$$
\hat{U}=\exp \left(-i \frac{k}{2 \hbar} \sum_{i=1}^{M} \hat{p}_{i}^{2}\right),
$$

in terms of which one has

$$
\begin{aligned}
\hat{q}_{n+1}^{a} & =U^{\dagger} \hat{q}_{n}^{a} U \\
& =\hat{q}_{n}^{a}+\sum_{b=1}^{M} \frac{k}{2 \hbar}\left[\left(p_{n}^{b}\right)^{2}, q_{n}^{a}\right] \\
& =\hat{q}_{n}^{a}+k \hat{p}_{n}^{a} \Theta_{a M},
\end{aligned}
$$

so we see that we have recovered the classical equations as operatorial relations in the Heisenberg representation.

We now wish to define the physical space of states of the continuum theory. To do this we will use the projector technique,

$$
\hat{P} \equiv \lim _{R \rightarrow \infty} C_{R} \hat{U}^{R}
$$

with $C_{R}=\left(\frac{i R k}{2 \pi \hbar}\right)^{M / 2}$. Acting on the identity written as $I=\int d p^{1} \cdots d p^{N}\left|p^{1}, \ldots, p^{N}><p^{1}, \ldots, p^{N}\right|$ we have,

$$
\begin{aligned}
\hat{P} & =\hat{P} I=\int d p^{1} \cdots d p^{N} \lim _{R \rightarrow \infty} C_{R} \exp \left(-\frac{i R k}{2 \hbar} \sum_{a=1}^{M}\left(p^{a}\right)^{2}\right)\left|p^{1}, \ldots, p^{N}><p^{1}, \ldots, p^{N}\right| \\
& =\int d p^{M+1} \cdots d p^{N}\left|0, \ldots, p^{M+1}, \ldots, p^{N}><0, \ldots, p^{M+1}, \ldots, p^{N}\right| .
\end{aligned}
$$


We therefore see that the physical space is given by the square integrable functions depending on the variables $M+1$ to $N$. The physical inner product is therefore immediately induced by the kinematical inner product on the physical space of states.

We would like now to turn our attention to a quantization where we assume the continuum limit is not taken, i.e. we end up with a discrete quantum theory. Although this is not necessary in this example, it would be in examples where the continuum limit is problematic. It is therefore good to see how the discrete quantum theory can be viewed as a quantizations of the continuum theory, at least at certain scales. Since we have a conserved Hamiltonian if we choose an initial quantum state that has a small expectation value for the Hamiltonian, it will keep its small expectation value upon the unitary evolution defined by $U$. We will take advantage of working in the discrete theory to be able to measure variables that would not be observable in the continuum theory and in terms of them define a relational evolution that will approximate well the dynamics of the continuum theory.

We start by recalling one way in which the dynamics of models like this can be handled in the continuum via the use of "evolving constants" [15]. Given any function of the dynamical variables of the theory $f\left(q^{1}, \ldots, q^{N}, p^{1}, \ldots, p^{N}\right)$ one can define an "evolving constant". This is a function that depends on a given number of real parameters $f\left(T^{1}, \ldots, T^{M}, q^{M+1}, \ldots, q^{N}, p^{M+1}, \ldots, p^{N}\right)$ and that coincides with the quantity of interest on the constraint surface when the partial observables $q^{1}, \ldots, q^{M}$ take the values $T^{1}, \ldots, T^{M}$. The "evolving constant" is a physical observable of the theory, that is, it has vanishing Poisson brackets with the constraints. To determine the dynamics of the theory is to determine what is the value of the quantity of interest $f$ for a given value of the parameters $T^{1}, \ldots, T^{M}$. A potential drawback of this approach is that it requires to treat some of the variables of the problem as classical variables, something that may not appear very natural in certain regimes where all variables should exhibit quantum mechanical behaviors.

We now go back to the discrete theory. Since the theory has no constraints we can work in the kinematical space and all variables will be quantum mechanical in nature. We wish to describe the evolution relationally, defining conditional probabilities (see for instance [16]). The physical variable we will consider is $\hat{F} \equiv \hat{q}^{1} \hat{p}^{N}$. This operator coincides with the angular momentum $L_{1 N}=\hat{q}^{1} \hat{p}^{N}-\hat{q}^{N} \hat{p}^{1}$ evaluated on the constraint surface. We now need to choose a "clock". Here we need to be careful. If we choose $q^{1}, \ldots, q^{M}$ as "clock" variables the problem that may arise is that if one makes a very precise measurement of one of these variables would imply a large change in the expectation value of the Hamiltonian that would lead to lose the regime close to the continuum. This is understandable, in a theory with fundamental discreteness there has to be a limit as to how accurate a measurement one can make. For instance, this would be akin to claiming that in a discrete theory of quantum gravity one cannot measure lengths shorter than the Planck length. It should be recalled that this is also a rather artificial limitation. If one is dealing with a realistic theory that includes measuring devices in it, since the evolution is unitary, one is guaranteed to remain in the regime one chooses irrespective of what measurements are made with the devices the theory incorporates. One can only break the regime if one introduces measuring devices that are external to the theory. In the particular example we are considering the theory is too simple to encompass measuring devices within it. We are therefore forced to consider external measurement devices and therefore we need to limit their interactions with the model to respect the choice of initial conditions within the regime of being close to the continuum.

Taking the last point into account, the projector for a clock reading a value $\left(q^{1}, \ldots q^{M}\right)$, where we understand that each variable takes a value within an interval $\Delta q$ centered at each $q^{a}$, is

$$
P_{q}=\int_{\Delta} d q^{1} \cdots d q^{M} \int d p^{M+1} \cdots d p^{N}\left|q^{1}, \ldots, q^{M}, p^{M+1}, \ldots, p^{N}><q^{1}, \ldots, q^{M}, p^{M+1}, \ldots, p^{N}\right|
$$

and the projector for a measurement of $\hat{F}$ to be in an interval $\left(f_{1}, f_{2}\right)$ is,

$$
P_{F}=\int d q^{1} \cdots d q^{M} d p^{M+1} \cdots d p^{N}\left|q^{1}, \ldots, q^{M}, p^{M+1}, \ldots, p^{N}><q^{1}, \ldots, q^{M}, p^{M+1}, \ldots, p^{N}\right| \theta\left(f_{1} / q^{1}, p^{N}, f_{2} / q^{1}\right)
$$

where $\theta(a, b, c)=1$ if $a \leq b \leq c$ and zero otherwise.

The projector for a simultaneous measurement of the clock and $\hat{F}$ therefore is,

$$
P_{q} P_{F}=\int_{\Delta} d q^{1} \cdots d q^{M} \int d p^{M+1} \cdots d p^{N}\left|q^{1}, \ldots, q^{M}, p^{M+1}, \ldots, p^{N}><q^{1}, \ldots, q^{M}, p^{M+1}, \ldots, p^{N}\right| \theta\left(f_{1} / q^{1}, p^{N}, f_{2} / q^{1}\right) .
$$

Let us consider a state at instant $n=0$ of the form,

$$
\left|\psi_{0}>=\int d p^{1} \cdots d p^{N} c_{p^{1}, \ldots, p^{N}}\right| p^{1}, \ldots, p^{N}>
$$


therefore, upon evolution,

$$
\left|\psi_{n}>=\hat{U}^{n}\right| \psi_{0}>=\int d p^{1} \cdots d p^{N} c_{p^{1}, \ldots, p^{N}} \exp \left(-\frac{i k n}{2 \hbar} \sum_{i=1}^{M}\left(p^{i}\right)^{2}\right) \mid p^{1}, \ldots, p^{N}>.
$$

The conditional probability is given by $\mathcal{P}(F \mid q)=\left(\sum_{n}<\psi_{n}\left|P_{q} P_{F}\right| \psi_{n}>\right) /\left(\sum_{n}<\psi_{n}\left|P_{q}\right| \psi_{n}>\right)$ that is, the ratio of the probability that the clock measures $q$ and the system under study takes the value $F$ simultaneously and the probability that the clock measures $q[16]$. Explicitly,

$$
\mathcal{P}(F \mid q)=\frac{\sum_{n} \int_{\Delta} d q^{1} \cdots d q^{M} \int d p^{M+1} \cdots d p^{N} \theta\left(f_{1} / q^{1}, p^{N}, f_{2} / q^{1}\right)\left|D_{n}\left(q^{1}, \ldots, q^{M}, p^{M+1}, \ldots, p^{N}\right)\right|^{2}}{\sum_{n} \int_{\Delta} d q^{1} \cdots d q^{M} \int d p^{M+1} \cdots d p^{N}\left|D_{n}\left(q^{1}, \ldots, q^{M}, p^{M+1}, \ldots, p^{N}\right)\right|^{2}}
$$

where we have defined

$$
D_{n}\left(q^{1}, \ldots, q^{M}, p^{M+1}, \ldots, p^{N}\right) \equiv \int d p^{1} \cdots d p^{M} c_{p^{1}, \ldots, p^{N}} \prod_{j=1}^{M}<q^{j} \mid p^{j}>\exp \left(-\frac{i k n}{2 \hbar}\left(p^{j}\right)^{2}\right) .
$$

If we assume that the initial state at $n=0$ takes a tensorial product form (which means the "clock" is not interacting with the "system under study") $c_{p^{1}, \ldots, p^{N}}=a_{p^{1}, \ldots, p^{M}} b_{p^{M+1}, \ldots, p^{N}}$ we have

$$
\frac{\int_{\Delta} d q^{1} \cdots d q^{M} \int d p^{M+1} \cdots d p^{N} \theta\left(f_{1} / q^{1}, p^{N}, f_{2} / q^{1}\right)\left|b_{p^{M+1}, \ldots, p^{N}}\right|^{2} h\left(q^{1}, \ldots, q^{M}\right)}{\int_{\Delta} d q^{1} \cdots d q^{M} \int d p^{M+1} \cdots d p^{N}\left|b_{p^{M+1}, \ldots, p^{N}}\right|^{2} h\left(q^{1}, \ldots, q^{M}\right)}
$$

for the conditional probability. Here we have defined

$$
h\left(q^{1}, \ldots, q^{M}\right) \equiv \sum_{n}\left|\int d p^{1} \cdots d p^{M} a_{p^{1}, \ldots, p^{M}} \prod_{j=1}^{M}<q^{j}\right| p^{j}>\left.\exp \left(-\frac{i k n}{2 \hbar}\left(p^{j}\right)^{2}\right)\right|^{2} .
$$

Since we are interested in comparing the results of our approach with that of the evolving constant approach in the continuum, we will now take the continuum limit. It is given by, $a_{p^{1}, \ldots, p^{M}}=\delta\left(p^{1}\right) \cdots \delta\left(p^{M}\right)$ the $h\left(q^{1}, \ldots, q^{M}\right)$ become constants and the probability is

$$
\mathcal{P}(F \mid q)=\frac{\int_{\Delta} d q^{1} \int d p^{M+1} \cdots d p^{N} \theta\left(f_{1} / q^{1}, p^{N}, f_{2} / q^{1}\right)\left|b\left(p^{M+1}, \ldots, p^{N}\right)\right|^{2}}{\Delta \int d p^{M+1} \cdots d p^{N}\left|b\left(p^{M+1}, \ldots, p^{N}\right)\right|^{2}}
$$

This completes the calculation in the relational picture.

Let us now revert, for comparison, to the "evolving constant" picture. To do this we need to construct a state of the physical space of the continuum theory such that it yields the same expectation value for the observables that $\mid \psi_{0}>$. It is given by a density matrix,

$$
\rho_{\text {phys }}=\left.\operatorname{Tr}\right|_{q^{1} \ldots q^{M}}\left|\psi_{0}><\psi_{0}\right| \text {. }
$$

The physical quantity to measure is $\hat{F} \equiv q^{1} \hat{p}^{N}$ (where $q^{1}$ is viewed as a c-number and therefore this quantity is an observable). The probability of getting a value in the interval $\left(f_{1}, f_{2}\right)$ is

$$
\begin{aligned}
\mathcal{P} & \equiv \frac{\operatorname{Tr}\left(\int_{\Delta} d q^{1} \int d p^{1} \cdots d p^{N}\left|p^{1}, \ldots, p^{N}><p^{1}, \ldots, p^{N}\right| \theta\left(f_{1} / q^{1}, p^{N}, f_{2} / q^{1}\right) \rho_{\text {phys }}\right)}{\Delta \operatorname{Tr}\left(\rho_{\text {phys }}\right)} \\
& =\frac{\int_{\Delta} d q^{1} \int d p^{M+1} \cdots d p^{N} \theta\left(f_{1} / q^{1}, p^{N}, f_{2} / q^{1}\right)\left|b\left(p^{M+1}, \ldots, p^{N}\right)\right|^{2}}{\Delta \int d p^{M+1} \cdots d p^{N}\left|b\left(p^{M+1}, \ldots, p^{N}\right)\right|^{2}}
\end{aligned}
$$

and in the case in which the state is a tensor product of the form $c_{p^{1}, \ldots, p^{N}}=a_{p^{1}, \ldots, p^{M}} b_{p^{M+1}, \ldots, p^{N}}$ this probability indeed coincides with (61).

We have therefore seen how one can work in the discrete theory to introduce a relational notion of time in the quantization of the discrete theory and how the physical predictions of this picture coincide with those of the "evolving constants" picture in the continuum theory if one takes the continuum limit. The agreement is qualified in the sense that we cannot carry out measurements of arbitrary precision of "classical" parameter of the evolving constant. This is to be expected in a treatment where all variables are treated quantum mechanically in an equal footing without singling out one to behave classically. 


\section{EXAMPLE 3: A SYSTEM WITH COMPACT GAUGE GROUP AND CONTINUUM LIMIT}

Here we will consider a system with non-Abelian constraints that form a Lie algebra. The model is given by the same phase space as in the previous section restricted to three configuration space dimensions. We consider a mechanical system with configuration manifold $R^{3}$, coordinatized by $q^{i}, i=1,2,3$ and 3 non-commuting constraints

$$
C^{i}=L^{i} \equiv \epsilon^{i j k} q^{j} p^{k} \quad i=1,2,3
$$

where the $p^{i}$ 's are the momenta conjugate to the $q^{i}$ 's and we assume Einstein's summation convention on repeated indices. Notice that the three constraints are not independent. Vanishing angular momentum is equivalent to requiring $q^{i}=\lambda p^{i}$ with $\lambda$ an arbitrary number, which implies two conditions between the phase space variables. The constraint surface is four dimensional and the system will have two independent observables. In our approach we could choose to construct the Hamiltonian starting from two independent constraints or one could choose to use a more symmetric, yet redundant, form of the constraints. We will choose the latter. We introduce a canonical evolution through a Hamiltonian,

$$
H=\sum_{i=1}^{3} \frac{\left(L^{i}\right)^{2}}{2 k},
$$

where $k$ is a constant with units of action. The discrete evolution equations are,

$$
\begin{aligned}
& q_{n+1}^{i}=q_{n}^{i}+\epsilon_{i j k} \frac{q^{k} L^{j}}{k}+\epsilon_{i j k} \epsilon_{j m n} q^{m} \frac{L^{n} L^{k}}{k^{2}}+\ldots \\
& p_{n+1}^{i}=p_{n}^{i}+\epsilon_{i j k} \frac{p^{k} L^{j}}{k}+\epsilon_{i j k} \epsilon_{j m n} p^{m} \frac{L^{n} L^{k}}{k^{2}}+\ldots
\end{aligned}
$$

The continuum limit is obtained setting $H=\delta^{2} / 2$ and defining $\lambda^{i}=L^{i} /(\delta \sqrt{k})$ we have, taking the limit as explained before,

$$
\begin{aligned}
& \dot{q}^{i}=\epsilon_{i j k} \frac{q^{k} \lambda^{j}}{\sqrt{k}}, \\
& \dot{p}^{i}=\epsilon_{i j k} \frac{p^{k} \lambda^{j}}{\sqrt{k}} .
\end{aligned}
$$

We note that the components $L^{i}$ are constants of the motion and therefore $\lambda^{i}$ are constant. There exist three further constants $q \cdot q=q^{i} q^{i}, p \cdot p=p^{i} p^{i}, q \cdot p=q^{i} p^{i}$. These are not independent since $L^{2}-\left((q \cdot q)(p \cdot p)-(q \cdot p)^{2}\right)=0$. One therefore has five independent constants of the motion of the discrete theory. In the continuum limit the $L^{i}$ 's vanish and one is left with two independent constants of motion, for instance $q \cdot q$ and $q \cdot p$. In the continuum theory, the trajectories are arbitrary trajectories on a sphere. In the discrete theory, when one takes the continuum limit one obtains trajectories that correspond to arbitrary circumferences on the sphere, since the $\lambda^{i}$ 's are constant. The constraint surface is therefore completely covered, but not all orbits of the continuum theory. This however, does not cause problems since we can recover all physical information of relevance with the trajectories we get.

To quantize the model, we construct the same kinematical space as in the previous example (restricted to a three dimensional configuration space), and immediately construct the unitary operator,

$$
\hat{U}=\exp \left(-i \frac{\hat{L}^{2}}{2 k \hbar}\right),
$$

which recovers as operatorial relations the classical discrete evolution equations (up to terms of order $\hbar$ ) so the correspondence principle is satisfied.

To compute the projector we use the formula (29) and, in terms of the a basis labeled by the radial and angular momentum eigenvalues,

$$
\hat{P}\left|n, \ell, m>=\delta_{\ell 0}\right| n, \ell, m>=\delta_{\ell 0} \mid n, 0,0>
$$

which can be rewritten as,

$$
\hat{P}=\sum_{n=0}^{\infty}|n, 0,0><0,0, n| .
$$


The continuum limit is therefore trivially achieved. The physical space is the space of zero angular momentum and there the constraints of the continuum theory are automatically satisfied. We therefore recover the traditional Dirac quantization. The physical space is given by the square integrable functions depending on the radial variable $|q|$. The physical inner product is therefore immediately induced by the kinematical inner product on the physical space of states.

We will not work out in detail the quantization in which the continuum limit is not taken, since it runs along similar lines as what we discussed in the previous section. The only thing to notice is that the current model would have a "time variable" consisting of two angles in the sphere. Again, one has restrictions on the accuracy with which one can measure angles so the approximation of the continuum theory is not lost.

\section{EXAMPLE 4: A SYSTEM WITH NON-COMPACT GAUGE GROUP}

Here we will consider a system with non-Abelian constraints that form a Lie algebra that is non-compact. More specifically, the model is associated with the $S O(2,1)$ group. It has the same phase space as in the previous section, except that the metric will be given by $\operatorname{diag}(-1,1,1)$. The configuration variables are $q^{i}$ with $i=0,1,2$ and the conjugate momenta are $p_{i}$. The constraints are

$$
C_{i}=L_{i} \equiv \epsilon_{i j}{ }^{k} q^{j} p_{k} \quad i=0,1,2 \quad,
$$

we assume Einstein's summation convention on repeated indices. Here one has to be careful about upper and lower indices since the metric is non-trivial. As in the previous example we notice that the three constraints are not independent. The constraint surface is four dimensional and the system will have two independent observables. In our approach we could choose to construct the Hamiltonian starting from the two independent constraints or one could choose to use a more symmetric, yet redundant, form of the constraints. We will choose the latter. We introduce a canonical evolution through a Hamiltonian,

$$
H=\frac{L_{i} L^{i}+2 L_{0}^{2}}{2 k},
$$

where $L$ is a constant with units of action. The discrete evolution equations are,

$$
\begin{aligned}
& q_{n+1}^{i}=q_{n}^{i}+\epsilon_{j k}^{i} \frac{q^{k} L^{j}}{k}-2 \epsilon_{0 k}^{i} \frac{q^{k} L^{0}}{k}+\ldots \\
& p_{n+1}^{i}=p_{n}^{i}+\epsilon_{j k}^{i} \frac{p^{k} L^{j}}{k}-2 \epsilon_{0 k}^{i} \frac{p^{k} L^{0}}{k}+\ldots
\end{aligned}
$$

The continuum limit is obtained setting $H=\delta^{2} / 2$. Defining $\lambda^{i}=L^{i} /(\delta \sqrt{k})$ for $i=1,2$ and $\lambda^{0}=-L^{0} /(\delta \sqrt{k})$ we have, taking the limit as explained before,

$$
\begin{aligned}
& \dot{q}^{i}=\epsilon_{j k}^{i} \frac{q^{k} \lambda^{j}}{\sqrt{k}}, \\
& \dot{p}^{i}=\epsilon_{j k}^{i} \frac{p^{k} \lambda^{j}}{\sqrt{k}} .
\end{aligned}
$$

We note that although $L_{0}$ is a constant of the motion, the other components are not. However $L_{1}^{2}+L_{2}^{2}$ is a constant, so $L_{1}$ and $L_{2}$ rotate around $L_{0}$ throughout the evolution. There exist three further constants $q \cdot q=q^{i} q_{i}, p \cdot p=p^{i} p_{i}$ $q \cdot p=q^{i} p_{i}$. These are not independent since $L \cdot L-\left((q \cdot q)(p \cdot p)-(q \cdot p)^{2}\right)=0$. One therefore has four independent constants of the motion of the discrete theory. In the continuum limit two of these vanish and one is left with two constants of motion, for instance $q \cdot q$ and $q \cdot p$. In the continuum theory, the trajectories are arbitrary trajectories on a two hyperboloids, one space-like and one time-like. In the discrete theory, when one takes the continuum limit one obtains trajectories that correspond to particular choices of the Lagrange multipliers, depending on the initial conditions chosen.

The quantization of this model (and similar ones) is known to be problematic [17, 18, 19]. The core of the problem is that if one promotes the constraints to operators on the usual Hilbert space, they do not have a vanishing eigenvalue. This can happen, but usually the resolution is to extend the Hilbert space by including an improper basis of eigenvectors. This can be done in this case, but one finds that the spectrum again does not contain zero. More specifically, the continuum spectrum has eigenvalues larger than $\hbar^{2} / 4$. One can find eigenvectors with eigenvalues smaller than $\hbar^{2} / 4$, but they do not arise as limit of functions of the Hilbert space, i.e. they cannot form part of an 
improper basis of the Hilbert space. One can adopt the point of view that nevertheless the eigenvectors with zero eigenvalue are the "physical space" of the theory, essentially abandoning the idea that the physical space arises as a suitable limit of the kinematical space. This was advocated by [17, 19]. From the point of view of our technique this is not satisfactory, since we wish to construct the physical space of states starting from the quantum kinematical space, taking a limit. As we mentioned before, lacking the zero eigenvalue in the spectrum of the Hamiltonian yields our prescription for the projector on the physical space useless. With this in mind, the most satisfactory solution is the one chosen in [18], where one chooses as physical space the eigenvectors that have $\hbar^{2} / 4$ as eigenvalue. Another attractive possibility in the discrete approach is not to take the continuum limit and retain a level of fundamental discreteness. This is very natural in a model where it is difficult to achieve a vanishing eigenvalue for the constraints, a natural minimum existing for their eigenvalues. As we argued before, such models can approximate the semiclassical physics of the theory of interest with some restrictions on the type of states considered. There can therefore be viewed as the best thing one can do in terms of having an underlying quantum theory for the model that approximates the classical physics of interest.

\section{GRAVITY IN THREE DIMENSIONS}

In this section we will follow closely the construction of Noui and Perez [20]. We will therefore not repeat all the details here. The difference will be that they construct the physical space of states using group averaging and we will use the projector using the technique we discussed earlier in this paper.

We start with three dimensional gravity in first order formalism. The space-time $\mathcal{M}$ is a three dimensional oriented smooth manifold and the action is simply given by

$$
S[e, \omega]=\int_{\mathcal{M}} \operatorname{Tr}[e \wedge F(\omega)]
$$

where $e$ is the triad, i.e. a Lie algebra valued 1-form, $F(\omega)$ is the curvature of the three dimensional connection $\omega$ and $\operatorname{Tr}$ denotes a Killing form on $g$. For simplicity we will concentrate on Riemannian gravity so the previous fields should be thought as defined on $S U(2)$ principal bundle over $\mathcal{M}$.

The phase space is parameterized by the pull back to $\Sigma$ of $\omega$ and $e$. In local coordinates we can express them in terms of the 2-dimensional connection $A_{a}^{i}$ and the triad field $E_{j}^{b}=\epsilon^{b c} e_{c}^{k} \eta_{j k}$ where $a=1,2$ are space coordinate indices and $i, j=1,2,3$ are $s u(2)$ indices. The symplectic structure is defined by

$$
\left\{A_{a}^{i}(x), E_{j}^{b}(y)\right\}=\delta_{a}^{b} \delta_{j}^{i} \delta^{(2)}(x, y) .
$$

Local symmetries of the theory are generated by the first class constraints $D_{b} E_{j}^{b} \simeq 0$ and $F_{a b}^{i}(A) \simeq 0$.

To quantize the theory we first find a representation of the basic variables in an auxiliary Hilbert space $\mathcal{H}_{\text {aux }}$. The basic functionals of the connection are represented by the set of holonomies along paths $\gamma \subset \Sigma$. Given a connection $A$ and a path $\gamma$, one defines the holonomy $h_{\gamma}[A]$ by

$$
h_{\gamma}[A]=P \exp \int_{\gamma} A .
$$

As for the triad, its associated basic variable is given by the smearing of $E$ along co-dimension 1 surfaces. One promotes these basic variables to operators acting on an auxiliary Hilbert space where constraints are represented. The physical Hilbert space is defined by those 'states' that are annihilated by the constraints. As these 'states' are not normalizable with respect to the auxiliary inner product they are not in $\mathcal{H}_{\text {aux }}$ and have to be regarded rather as distributional.

The auxiliary Hilbert space is defined by the Cauchy completion of the space of cylindrical functionals $C y l$, on the space of (generalized) connections $\overline{\mathcal{A}}$. The space $C y l$ is defined as follows: any element of $C y l, \Psi_{\Gamma, f}[A]$ is a functional of $A$ labeled by a finite graph $\Gamma \subset \Sigma$ and a continuous function $f: S U(2)^{N_{\ell}(\Gamma)} \rightarrow C$ where $N_{\ell}(\Gamma)$ is the number of links of the graph $\Gamma$. Such a functional is defined as follows

$$
\Psi_{\Gamma, f}[A]=f\left(h_{\gamma_{1}}[A], \cdots, h_{\gamma_{N_{\ell}(\Gamma)}}[A]\right)
$$

where $h_{\gamma_{i}}[A]$ is the holonomy along the link $\gamma_{i}$ of the graph $\Gamma$. If one considers a new graph $\Gamma^{\prime}$ such that $\Gamma \subset \Gamma^{\prime}$, then any cylindrical function $\Psi_{\Gamma, f}[A]$ trivially corresponds to a cylindrical function $\Psi_{\Gamma^{\prime}, f^{\prime}}[A]$. This space can be endowed with the well known Ashtekar-Lewandowski inner product. The Gauss constraint can be defined in terms of the 
basic variables introduced above. It generates gauge transformations whose action on $C y l$ transforms the holonomy as follows

$$
h_{\gamma}[A] \longmapsto g_{s} h_{\gamma}[A] g_{t}^{-1}
$$

where $g_{s}, g_{t} \in S U(2)$ are group elements associated to the source and target nodes of $\gamma$ respectively. The so-called kinematical Hilbert space $\mathcal{H}_{\text {kin }} \subset \mathcal{H}_{\text {aux }}$ is defined by the states in $\mathcal{H}_{\text {aux }}$ which are gauge invariant and hence in the kernel of the Gauss constraint.

A basis of gauge invariant functions can then be constructed by contracting the tensor product of representation matrices with $s u(2)$-invariant tensors or $s u(2)$-intertwiners. If we select an orthonormal basis of intertwiners $\iota_{n} \in$ $\operatorname{Inv}\left[j_{1} \otimes j_{2} \otimes \cdots \otimes j_{N_{\ell}}\right]$ where $n$ labels the elements of the basis we can write a basis of gauge invariant elements of $C y l$ called the spin network basis. Each spin network is labeled by a graph $\Gamma \subset \Sigma$, a set of spins $j_{\gamma}$ labeling links $\gamma$ of the graph $\Gamma$ and a set of intertwiners $\iota_{n}$ labeling nodes $n$ of the graph $\Gamma$, namely:

$$
s_{\Gamma,\left\{j_{\ell}\right\},\left\{\iota_{n}\right\}}[A]=\bigotimes_{n \in \Gamma} \iota_{n} \bigotimes_{\gamma \in \Gamma} \prod_{\gamma}^{j_{\gamma}}\left(h_{\gamma}[A]\right)
$$

In order to lighten notations, we will omit indices (the graph, representations and intertwiners) labeling spin-networks in the sequel. One can take the kinematical inner product of two spin networks $s, s^{\prime}$, which we will denote $\left\langle s \mid s^{\prime}\right\rangle$ by integrating expression (84) with the Ashtekar-Lewandowski measure.

Let us review the group averaging procedure to generate the projector, following closely the discussion of Noui and Perez. One starts with the formal expression

$$
P=" \prod_{x \in \Sigma} \delta(\hat{F}(A)) "=\int D[N] \exp \left(i \int_{\Sigma} \operatorname{Tr}[N \hat{F}(A)]\right),
$$

where $N \in s u(2)$. We now introduce a regularization of (85). We will give a definition of $P$ by providing a regularization of its matrix elements $\left\langle P s, s^{\prime}\right\rangle$ for any pair of spin network states $s, s^{\prime} \in \mathcal{H}_{\text {kin }}$. Let's denote by $\Gamma$ and $\Gamma^{\prime}$ the graphs on which $s$ and $s^{\prime}$ are defined respectively. We introduce an arbitrary cellular decomposition of $\Sigma$ denoted $\Sigma_{\epsilon}^{\Gamma^{\prime}}$, where $\epsilon \in R$, such that the graphs $\Gamma$ and $\Gamma^{\prime}$ are both contained in the graph defined by the union of 0-cells and 1-cells in $\Sigma_{\epsilon}^{\Gamma \Gamma^{\prime}}$ and for each individual 2-cells (plaquette) $p$ there exist a ball $\mathcal{B}_{\epsilon}$ of radius $\epsilon$ - defined using the local topology - such that $p \subset \mathcal{B}_{\epsilon}$.

Consequently all 2-cells shrink to zero when $\epsilon \rightarrow 0$. We consider a local patch $U \subset \Sigma$ where we choose the cellular decomposition to be square with cells of coordinate length $\epsilon$. In that patch, the integral in the exponential in (85) can be written as a Riemann sum

$$
F[N]=\int_{U} \operatorname{Tr}[N F(A)]=\lim _{\epsilon \rightarrow 0} \sum_{p^{i}} \epsilon^{2} \operatorname{Tr}\left[N_{p^{i}} F_{p^{i}}\right],
$$

where $p^{i}$ labels the $i^{t h}$ plaquette and $N_{p^{i}} \in s u(2)$ and $F_{p^{i}} \in s u(2)$ are values of $N^{j} \tau_{j}$ and $\tau_{j} \epsilon^{a b} F_{a b}^{j}[A]$ at some interior point of the plaquette $p^{i}$ and $\epsilon^{a b}$ is the Levi-Civita tensor. The basic observation is that the holonomy $U_{p^{i}} \in S U(2)$ around the plaquette $p^{i}$ can be written as

$$
U_{p^{i}}[A]=\mathbb{1}+\epsilon^{2} F_{p^{i}}(A)+\mathcal{O}\left(\epsilon^{2}\right)
$$

which implies

$$
F[N]=\int_{U} \operatorname{Tr}[N F(A)]=\lim _{\epsilon \rightarrow 0} \sum_{p^{i}} \operatorname{Tr}\left[N_{p^{i}} U_{p^{i}}[A]\right],
$$

where the $\operatorname{Tr}$ in the right hand side is taken in the fundamental representation. Notice that the explicit dependence on the regulator $\epsilon$ has dropped out of the sum on the r.h.s., a sign that we should be able to remove the regulator upon quantization. With all this it is natural to write the following formal expression for the generalized projection operator,

$$
\begin{aligned}
<P s, s^{\prime}> & =\lim _{\epsilon \rightarrow 0}<\prod_{p^{i}} \int d N_{p^{i}} \exp \left(i \operatorname{Tr}\left[N_{p^{i}} \hat{U}_{p^{i}}\right]\right) s, s^{\prime}> \\
& =\lim _{\epsilon \rightarrow 0}<\prod_{p^{i}} \delta\left(U_{p^{i}}\right) s, s^{\prime}>
\end{aligned}
$$


where the last equality follows from direct integration over $N_{p^{i}}$ at the classical level; $\delta(U)$ being the distribution such that $\int d g f(g) \delta(g)=f(1)$ for $f \in \mathcal{L}^{2}(S U(2))$. We can write $\delta(U)$ as a sum over unitary irreducible representations of $S U(2)$, namely $\delta\left(U_{p^{i}}\right)=\sum_{j}(2 j+1) \chi_{j}\left(U_{p^{i}}\right)$, where $\chi_{j}(U)$ is the trace of the $j$-representation matrix of $U \in S U(2)$. It is therefore straightforward to evaluate explicitly the action of the projector.

We now proceed to construct the projector using our technique. We construct a Hamiltonian

$$
H=\sum_{p} F_{p}^{i} F_{p}^{i}
$$

where $F_{p}^{i} \tau^{i}$ is the element of the $S U(2)$ algebra such that exponentiated gives the holonomy around the plaquette $p$ ( $\tau^{i}$ are the Pauli matrices), $U(p)=\exp \left(i F_{p}\right)$. We now proceed to construct the projector as usual,

$$
\mathcal{P}=\lim _{\lambda \rightarrow \infty}\left(\frac{i \lambda}{\pi}\right)^{3 / 2} e^{(-i \lambda H)} .
$$

If this expression is to agree with the projector computed by Noui and Perez, one has to have that,

$$
\lim _{\lambda \rightarrow \infty} \int\left(\prod_{p} d g_{l}\right) f\left(g_{p_{1}}, g_{p_{2}}, \ldots\right) \mathcal{P}_{\lambda}=\int\left(\prod_{p} d g_{l}\right) f\left(g_{p_{1}}, g_{p_{2}}, \ldots\right) \prod_{p} \delta\left(g_{p}\right) .
$$

In order to prove this identity we start by introducing a parameterization of the $S U(2)$ group [21],

$$
U=\left(\begin{array}{cc}
a & b \\
-b^{*} & a^{*}
\end{array}\right)
$$

where

$$
\begin{aligned}
a & ==\cos \left(\frac{\omega}{2}\right)-i \sin \left(\frac{\omega}{2}\right) \cos \theta, \\
b & ==-i \sin \left(\frac{\omega}{2}\right) \sin \theta e^{i \varphi} .
\end{aligned}
$$

One then has that

$$
\int d g=4 \int_{0}^{\pi} \sin ^{2}\left(\frac{\omega}{2}\right) d \omega \int_{0}^{\pi} \sin \theta d \theta \int_{0}^{2 \pi} d \varphi
$$

and

$$
\chi^{j}(\omega)=\frac{\sin \left(\frac{(2 j+1) \omega}{2}\right)}{\sin \left(\frac{\omega}{2}\right)} .
$$

And we see that $\omega=0$ corresponds to $a=1, b=0$. The $F$ 's take the form,

$$
\begin{aligned}
& F^{1}=-\left(\frac{\omega}{2}\right) \sin \theta \cos \varphi, \\
& F^{2}=-\left(\frac{\omega}{2}\right) \sin \theta \sin \varphi, \\
& F^{3}=-\left(\frac{\omega}{2}\right) \cos \theta .
\end{aligned}
$$

From here we can compute $H$,

$$
H=\sum_{p} \omega_{p}^{2}
$$

and the projector can be written as,

$$
\mathcal{P}=\lim _{\lambda \rightarrow \infty} \prod_{p}\left(\frac{i \lambda}{\pi}\right)^{3 / 2} e^{\left(-i \lambda \omega_{p}^{2}\right)}
$$


To complete the proof we would like to show that

$$
\delta\left(g_{p}\right)=\frac{1}{4 \pi} \frac{d^{2}}{d \omega^{2}} \delta(\omega) \equiv \delta\left(g_{p}(\omega, \theta, \varphi)\right),
$$

which can be shown by noticing that

$$
\begin{aligned}
\int d g_{p} f\left(g_{p}\right) \delta\left(g_{p}(\omega, \theta, \varphi)\right) & =2 \int_{-\pi}^{\pi} d \omega \int d \Omega \sin ^{2}\left(\frac{\omega}{2}\right) f\left(g_{p}(\omega, \theta, \varphi)\right) \frac{1}{4 \pi} \frac{d^{2}}{d \omega^{2}} \delta(\omega) \\
& =2 \int_{-\pi}^{\pi} d \omega \sin ^{2}\left(\frac{\omega}{2}\right) f\left(g_{p}(\omega, \theta, \varphi)\right) \frac{d^{2}}{d \omega^{2}} \delta(\omega) \\
& =2 \int_{-\pi}^{\pi} d \omega f\left(g_{p}(\omega, \theta, \varphi)\right) \delta(\omega) \frac{d^{2}}{d \omega^{2}} \sin ^{2}\left(\frac{\omega}{2}\right) \\
& =\int_{-\pi}^{\pi} d \omega f\left(g_{p}(\omega, \theta, \varphi)\right) \delta(\omega) \cos \omega=f(\mathbb{1}) .
\end{aligned}
$$

Finally, using the following Dirac identity for distributions,

$$
\sqrt{\frac{i \lambda}{\pi}} e^{-i \lambda \omega_{p}^{2}}=\sum_{j=0}^{\infty}\left(\frac{-i}{2 \lambda}\right)^{j} \frac{1}{(2 j) ! !} \delta^{(2 j)}\left(\omega_{p}\right),
$$

where $\delta^{(2 j)}$ is the $(2 j)$-th derivative of the Dirac delta. Inserting this expression in (100) and using (102) we prove the identity (90) which implied that the projector we constructed with our technique agrees with the one obtained via group averaging by Noui and Perez.

\section{CONCLUSIONS}

We have introduced a new approach to the quantization of totally constrained systems and worked out several examples. One reaches two conclusions. On one hand, one can use the technique to construct in a well defined way the physical space of states of the totally constrained theory of interest. Of special appeal is the fact that one does it without the need of promoting the constraints to quantum operators, which in several situations is known to be problematic. In particular one does not have to deal with the problem of reproducing the quantum constraint algebra. In examples we have shown that the results coincide with those of the group averaging procedure, where the latter exists. Moreover one has a correspondence principle in that one needs to reproduce the classical equations of motion as quantum operatorial relations that offers guidance in the intermediate steps of the process. The technique therefore has aspects that are quite distinct from conventional ones, especially when one treats field theories.

On the other hand the technique can be viewed as a new paradigm for dealing with cases where the continuum theory does not exist. In those cases one builds discrete theories where one can introduce a relational notion of time and compute probabilities and expectation values. In examples we have shown that if one is able to take the continuum limit, one reproduces predictions of the evolving constants approach and therefore recovers all the physics in the theory. But we have also shown in examples where the continuum limit does not exist that one can define semiclassical regimes that reproduce, at certain scales, the classical results of the theory of interest. Although we do not yet know if the continuum limit exists in the case of general relativity, this point of view would agree with popular beliefs in that case: one would have a fundamental, discrete theory that reproduces, at large scales, general relativity even if the quantum theory strictly does not exist in the continuum limit.

Finally, the uniform discretizations also limit importantly the usual ambiguities that appear when one discretizes theories and may even be useful in the classical modeling of constrained theories. Future steps will require to test the technique in cases of increasing complexity in the gravitational case. We have shown that in the cosmological case one reproduces the results of loop quantum cosmology and the technique may allow to explore some of the attractive features of the latter in more complex models in the near future.

\section{Acknowledgments}

We wish to thank Sebastián Torterolo for help with the cosmology section. This work was supported in part by grants NSF-PHY-0244335, NSF-PHY-0554793, CCT-LSU, FQXi, Pedeciba, DID-USB grant GID-30 and Fonacit 
grant G-2001000712 and the Horace Hearne Jr. Institute for Theoretical Physics.

[1] C. Rovelli. Living Rev. Rel. 1 (1998), 1. gr-qc/9710008; T. Thiemann. Lect. Notes Phys. 631 (2003), $41-135$. gr-qc/0210094; A. Ashtekar and J. Lewandowski Class. Quant. Grav. 21 (2004), R53. gr-qc/0404018; L. Smolin. "An invitation to loop quantum gravity" hep-th/0408048

[2] A. Ashtekar and C.J. Isham Class. Quantum Grav. 9 (1992), 1433. hep-th/9202053; A. Ashtekar and J. Lewandowski. "Representation theory of analytic holonomy $C^{\star}$ algebras" In Knots and Quantum Gravity, J. Baez (ed.), (Oxford University Press, Oxford 1994); A. Ashtekar, J. Lewandowski, D. Marolf, J. Mourão and T. Journ. Math. Phys. 36 (1995), 6456-6493. gr-qc/9504018

[3] H. Sahlmann "Some comments on the representation theory of the algebra underlying loop quantum gravity" gr-qc/0207111; A. Okolow and J. Lewandowski Class. Quant. Grav. 20 (2003), 3543-3568 [gr-qc/03027112]; A. Okolow and J. Lewandowski Class. Quant. Grav. 22 (2005), 657-680. gr-qc/0405119; H. Sahlmann and T. Thiemann "On the superselection theory of the Weyl algebra for diffeomorphism invariant quantum gauge theories." gr-qc/0302090; H. Sahlmann and T. Thiemann Class. Quant. Grav. 23 (2006), 4453-4472 gr-qc/0303074; J. Lewandowski, A. Okolow, H. Sahlmann and T. Thiemann Comm. Math. Phys. (accepted) gr-qc/0504147

[4] K. Giesel and T. Thiemann, "Algebraic quantum gravity I-III" arXiv:gr-qc/0607099 0607100,0607101.

[5] T. Thiemann. Class. Quant. Grav. 23 (2006), 2211-2248 gr-qc/0305080; Class. Quant.Grav. 23 (2006), 2249-2266 gr-qc/0510011; B. Dittrich and T. Thiemann. Class. Quant. Grav. 23 (2006), 1025-1066 gr-qc/0411138; Class. Quant. Grav. 23 (2006), 1067-1088 gr-qc/0411139]; Class. Quant. Grav. 23 (2006), 1089-1120 gr-qc/0411140; Class. Quant. Grav. 23 (2006), 1121-1142 gr-qc/0411141]; Class. Quant. Grav. 23 (2006), 1143-1162 gr-qc/0411142.

[6] J. R. Klauder, Nucl. Phys. B 547, 397 (1999) arXiv:hep-th/9901010.

[7] R. Gambini and J. Pullin, "Classical and quantum general relativity: A new paradigm," Fifth Prize in the Gravity Research Foundation 2005 essay competition. Gen. Rel. Grav. 37, 1689 (2005) [Int. J. Mod. Phys. D 14, 2355 (2005) arXiv:gr-qc/0505052.

[8] R. Gambini and J. Pullin, "Consistent discretizations as a road to quantum gravity," to appear in "Approaches to quantum gravity", D. Oriti (editor), Cambridge University Press, Cambridge, UK arXiv:gr-qc/0512065.

[9] R. Gambini and J. Pullin, "Discrete space-time," arXiv:gr-qc/0505023 published in "100 years of relativity", Abhay Ashtekar (editor), World Scientific, Singapore (2006).

[10] M. Campiglia, C. Di Bartolo, R. Gambini and J. Pullin, "Uniform discretizations: A quantization procedure for totally constrained arXiv:gr-qc/0606121

[11] C. Di Bartolo, R. Gambini, R. Porto and J. Pullin, J. Math. Phys. 46, 012901 (2005) arXiv:gr-qc/0405131.

[12] A. Higuchi, Class. Quan. Grav. 8, 1983 (1991); A. Ashtekar, J. Lewandowski, D. Marolf, J. Mourao and T. Thiemann, "Quantization of diffeomorphism invariant theories of connections with local J. Math. Phys. 36, 6456 (1995) arXiv:gr-qc/9504018.

[13] A. Ashtekar, M. Bojowald and J. Lewandowski, Adv. Theor. Math. Phys. 7, 233 (2003) arXiv:gr-qc/0304074. A. Ashtekar, T. Pawlowski and P. Singh, Phys. Rev. Lett. 96, 141301 (2006) arXiv:gr-qc/0602086].

[14] T. Thiemann, Class. Quant. Grav. 15, 839 (1998) arXiv:gr-qc/9606089.

[15] C. Rovelli, Phys. Rev. D 43, 442 (1991).

[16] R. Gambini, R. Porto and J. Pullin, New J. Phys. 6, 45 (2004) arXiv:gr-qc/0402118.

[17] A. Gomberoff and D. Marolf, Int. J. Mod. Phys. D 8, 519 (1999) arXiv:gr-qc/9902069.

[18] B. Dittrich and T. Thiemann, Class. Quant. Grav. 23, 1089 (2006) arXiv:gr-qc/0411140].

[19] J. Louko and C. Rovelli, "Refined algebraic quantization in the oscillator representation of J. Math. Phys. 41, 132 (2000) arXiv:gr-qc/9907004.

[20] K. Noui and A. Perez, Class. Quant. Grav. 22, 1739 (2005) arXiv:gr-qc/0402110.

[21] D. A. Varshalovich A. N. Moskalev, V. K. Khersonskii, "Quantum theory of angular momentum", World Scientific, Singapore (1988). 